OPEN ACCESS

Edited by:

Cristina Has,

Albert-Ludwigs-Universität Freiburg,

Germany

Reviewed by:

Marian Dmochowski,

Poznan University of Medical

Sciences, Poland

Takashi Hashimoto,

Osaka University, Japan

*Correspondence:

Khalaf Kridin

dr_kridin@hotmail.com

Specialty section: This article was submitted to

Dermatology,

a section of the journal

Frontiers in Medicine

Received: 15 July 2018 Accepted: 03 September 2018

Published: 17 October 2018

Citation:

Kridin K and Bergman R (2018) The

Usefulness of Indirect

Immunofluorescence in Pemphigus and the Natural History of Patients With Initial False-Positive Results: A

Retrospective Cohort Study.

Front. Med. 5:266.

doi: 10.3389/fmed.2018.00266

\section{The Usefulness of Indirect} Immunofluorescence in Pemphigus and the Natural History of Patients With Initial False-Positive Results: A Retrospective Cohort Study

\author{
Khalaf Kridin $^{1 *}$ and Reuven Bergman ${ }^{1,2}$ \\ ${ }^{1}$ Department of Dermatology, Rambam Health Care Campus, Haifa, Israel, ${ }^{2}$ Rappaport Faculty of Medicine, Technion-Israel \\ Institute of Technology, Haifa, Israel
}

The specificity and the predictive values of indirect immunofluorescence (IIF) in real-life settings is yet to be firmly established. The natural history of patients with false-positive results has not been sufficiently elucidated. The primary aim of the current study is to evaluate the diagnostic value of IIF analysis on monkey esophagus in pemphigus, utilizing a large cohort arising from the real-life experience of a tertiary referral center. The secondary endpoint was to determine the clinical outcomes of patients with false-positive results. This was a retrospective cohort study including all patients who were tested for the presence of intercellular autoantibodies by IIF on monkey esophagus between 2000 and 2017. Overall, 770 sera from different individuals were tested by IIF microscopy. Of those, 176 patients had been diagnosed with pemphigus vulgaris (PV) and 29 patients with pemphigus foliaceus (PF). The sensitivity of this immunoassay was significantly higher for the diagnosis of PV (87.4\%; 95\% Cl, 81.5-91.9\%) as compared to PF (69.0\%; 95\% Cl, 49.2-84.7\%; $P=0.018)$. The specificity for the diagnosis of pemphigus was $93.5 \%$ (95\% Cl, 91.1-95.4\%). Patients with false-positive results $(n=37)$ were followed for a median duration of 5.3 years contributing 280.8 person-years. Thirty patients $(81.1 \%)$ were eventually diagnosed clinically and immunopathologically with subepidermal autoimmune bullous diseases, whereas the remaining patients (18.9\%) were diagnosed clinically and histologically with other inflammatory dermatoses, but none of them developed pemphigus during the follow-up duration. Of note, $7.0 \%(n=23)$ of all patients diagnosed with bullous pemphigoid (BP) in the same period $(n=328)$ were tested positive for IgG intercellular antibodies. Histopathological review of the biopsy specimens of these patients did not reveal acantholysis. In conclusion, the predictive value of negative test in IIF on monkey esophagus is particularly reliable to exclude a diagnosis of pemphigus. Individuals tested positive for intercellular antibodies without an initial overt pemphigus did not show an increased risk for developing pemphigus subsequently. A sizable fraction of patients with BP showed circulating intercellular autoantibodies by IIF, without a histopathological evidence for acantholysis.

Keywords: pemphigus, indirect immune fluorescence assay, monkey esophagus, pemphigus vulgaris, pemphigus foliaceus, false positive, sensitivity, specificity 


\section{INTRODUCTION}

Pemphigus is a rare, chronic, potentially life-threatening, autoimmune bullous disease of the skin and the mucous membranes. The two major subtypes of pemphigus are pemphigus vulgaris $(\mathrm{PV})$ and pemphigus foliaceus $(\mathrm{PF})$. The etiopathogenesis underlying the development of the disease is characterized by acantholysis and intraepidermal blister formation, resulting from IgG autoantibodies directed against desmoglein (Dsg) 3 (PV) and/or Dsg 1 (PF), two transmembrane desmosomal glycoproteins $(1,2)$.

An essential element of the diagnosis of pemphigus is the detection of circulating intercellular antibodies. Despite the detailed knowledge of pemphigus antigens and the development of increasing number of sensitive and specific assays for the detection of circulating autoantibodies, including Western blotting of cell-derived and recombinant forms of the target antigens, immunoprecipitation, and enzymelinked immunosorbent assay (ELISA), the diagnosis of pemphigus in most laboratories still relies chiefly upon indirect-immunofluorescence (IIF) technique. Although a universally sensitive substrate has not been established, IIF on monkey esophagus has been elucidated as the most sensitive screening test (3-5). The value of IIF titers in disease monitoring has been a subject of debate, with conflicting results throughout the years. Although early studies suggested that intercellular antibodies levels measured by IIF were a useful marker of disease activity (6-8), later studies concluded that IIF titers did not always correlate with the disease severity, and are not consistent enough to serve as a guide for therapy or for monitoring the disease activity $(9-12)$.

IIF is positive in approximately $70-90 \%$ of pemphigus patients but lacks the ability to differentiate definitively between $\mathrm{PV}$ and PF since both have IgG antibodies directed against keratinocyte cell surface $(4,13,14)$. The sensitivity of this assay varies according to the specificity of the epithelial substrate the sera are incubated with $(4,14-17)$. To the best of our knowledge, the specificity and the predictive values of this immunological assay was not estimated in real-life settings in the past. Unlike controlled trials examining the diagnostic features of this immunoassay under optimal setting in the absence of confounding factors, real life studies inform on the effectiveness of an analysis when performed in routine circumstances, by including all patients with initial suspicion of autoimmune bullous disease and not only patients and healthy control subjects. Furthermore, the natural history of patients with false-positive results is yet to be fully elucidated.

The aim of the current study is to evaluate the sensitivity, specificity and predictive values of IIF analysis on monkey esophagus utilizing a large cohort extracted from the reallife experience of a tertiary center throughout an extended duration. Our secondary endpoint was to determine the clinical outcomes and histological features of patients tested positive for intercellular antibodies without an initial diagnosis of pemphigus (false-positive patients).

\section{METHODS}

This was a retrospective cohort study that included all patients who were tested for the presence of intercellular autoantibodies by (IIF) in Rambam Health Care Campus, Haifa, Israel, throughout the years 2000-2017. The current study was approved by the institutional ethical board of our medical center.

\section{Patients and Case Definition}

The clinical and immunopathological criteria for the diagnosis of PV were: (1) presence of skin blisters and/or erosions on mucous membranes; (2) suprabasal intraepidermal acantholysis on histopathological examination of skin and/or mucosa; and (3) intraepidermal intercellular IgG and/or C3 deposits by direct immunofluorescence (DIF); or intercellular circulating antibodies demonstrated by using monkey esophagus and a standard IIF technique; or the presence of anti-Dsg $3 \pm$ anti-Dsg 1 autoantibodies, measured by ELISA (UROIMMUN Medizinische Labordiagnostika AG; Lübeck) or immunoblotting (on human dermal and epidermal extracts as antigenic substrate) (18).

The clinical and immunopathological criteria for the diagnosis of PF were: (1) presence of skin blisters or erosions; (2) lack of mucosal lesions; (3) intraepidermal acantholysis compatible with PF on histopathological examination; and (4) intraepidermal intercellular IgG and/or C3 deposits by DIF; or intercellular circulating antibodies demonstrated by a standard IIF technique; or presence of anti-Dsg 1 autoantibodies, with lack of anti-Dsg 3 autoantibodies, measured by ELISA or immunoblotting (18).

The differentiation between the different phenotypes of PV (isolated mucosal, isolated cutaneous, or mucocutaneous) was grounded on the clinical and endoscopic (in cases of laryngeal involvement) presentation at the onset of the disease, without referring to serological analyses to ascertain that those with the isolated mucosal disease had only anti-Dsg 3 antibodies, and those with mucocutaneous had both anti-Dsg 3 and anti-Dsg 1 antibodies.

\section{Indirect Immunofluorescence}

All sera were tested in serial dilution for intercellular antibodies by IIF. IIF assay using monkey esophagus as the substrate was performed according to a standard technique (18). Sera samples were incubated with monkey esophagus, and fluoresceinlabeled goat anti-human IgG sera (Tago, Camarillo, CA) were added subsequently. Each serum sample was examined under fluorescence microscopy. Serum samples were considered to be positive if they stained epidermal intercellular spaces at a titer of $\geq 20$.

\section{Histopathology}

Hematoxylin-eosin stained cutaneous biopsies of patients with false-positive results were re-examined for evidence of acantholysis.

\section{Statistical Analysis}

All continuous parameters were expressed as mean values \pm standard deviation (SD). Categorical variables were expressed as proportions. Comparisons of percentages between different 
patient groups were carried out using the Chi-square test. To determine the sensitivity and specificity of the IIF immunoassay, receiver operating curves (ROC) were analyzed. For sample proportions, 95\% confidence interval (CI) was computed using the Clopper-Pearson exact binomial proportion interval method as fitting. CIs for the likelihood ratios were calculated using the "Log method." CIs for the predictive values were the standard logit CIs given by Mercaldo et al. (19). Figures with $P$-values $<$ 0.05 were considered statistically significant. Statistical analysis was performed using IBM SPSS statistics software, version 23 (IBM Corp, Armonk, New York, USA) and MedCalc Statistical Software (version 16.4.3, MedCalc Software, Ostend, Belgium).

\section{RESULTS}

\section{Clinical and Demographic Characteristics of the Study Participants}

Overall, 770 sera from different individuals were tested for the presence of intercellular autoantibodies by IIF microscopy between the years 2000 and 2017. Of those, 176 patients had been diagnosed with PV and 29 patients with PF. IIF analysis was performed on the sera of 174 (98.9\%) patients diagnosed with PV and all patients with PF before the initiation of any immunosuppressive therapy. With regard to patients with PV, the most frequent clinical phenotype was mucocutaneous $(n=106$; $60.2 \%)$, followed by isolated mucosal disease $(n=63 ; 35.8 \%)$ and isolated cutaneous disease $(n=7 ; 4.0 \%)$. The demographic and clinical characteristics of the patients with pemphigus included in the analysis are demonstrated in Table $\mathbf{1 .}$

\section{The Diagnostic Value of IIF on Monkey Esophagus}

Of the 174 sera from PV patients tested by IIF, 152 were positive for intercellular antibodies against monkey esophagus, corresponding to a sensitivity of $87.4 \%$ (95\% CI, 81.5-91.9\%; Table 2). When PV patients were divided according to the clinical phenotype, IIF sensitivity was comparable for those with isolated mucosal (87.1\%; 95\% CI, 76.2-94.3\%) and mucocutaneous phenotypes (87.6\%; 95\% CI, 79.8-93.2\%; $P=0.925)$. Patients

TABLE 1 | Demographic and clinical characteristics of patients with pemphigus whose sera were tested by indirect immunofluorescence microscopy.

\begin{tabular}{lcc}
\hline & $\begin{array}{c}\text { Pemphigus } \\
\text { vulgaris }(\boldsymbol{n}=\mathbf{1 7 4})\end{array}$ & $\begin{array}{c}\text { Pemphigus foliaceus } \\
(\boldsymbol{n}=\mathbf{2 9})\end{array}$ \\
\hline AGE AT DIAGNOSIS & & \\
Mean \pm SD & $55.5 \pm 15.3$ & $57.1 \pm 21.4$ \\
Median (range) & $55(20-90)$ & $63(20-87)$ \\
$\%$ female $(\mathrm{n})$ & $65.9 \%(116)$ & $34.5 \%(10)$ \\
CLINICAL PHENOTYPE, \% (N) & $\mathrm{NA}$ \\
$\quad$ Isolated mucosal & $35.6(62)$ & $\mathrm{NA}$ \\
Mucocutaneous & $60.4(105)$ & $\mathrm{NA}$ \\
Isolated cutaneous & $4.0(7)$ &
\end{tabular}

n, number; $S D$, standard deviation. with isolated cutaneous disease had lower sensitivity (57.1\%; 95\% CI, 18.4-90.1\%), but the small size of this subgroup $(n=7)$ hinders drawing meaningful comparisons. The sensitivity of IIF on monkey esophagus in PF patients was only 69.0\% (95\% CI, 49.2-84.7\%; Table 2). Taken together, the sensitivity of this immunoassay was significantly higher for the diagnosis of $\mathrm{PV}$ as compared to that of PF $(P=0.018)$.

Overall, 770 patients were tested by IIF microscopy, including $567(73.6 \%)$ patients who did not have an established diagnosis of pemphigus. Of those, $37(6.5 \%)$ were tested positive for intercellular antibodies by IIF. Altogether, the specificity of this immunoassay for the diagnosis of pemphigus was as high as 93.5\% (95\% CI, 91.1-95.4\%).

The positive predictive value (PPV) of IIF on monkey esophagus for the diagnosis of PV was 80.4\% (95\% CI, 75.0$84.9 \%$ ) and the negative predictive value (NPV) was $96.0 \%$ (95\% CI, 94.2-97.3\%). The positive likelihood ratio (PLR) was 13.4 (95\% CI, 9.8-18.4), while the negative likelihood ratio (NLR) was 0.14 (95\% CI, 0.1-0.2). With regard to PF, the PPV of this assay was $35.1 \%$ (95\% CI, 26.7-44.5\%), whereas the NPV was 98.3\% (95\% CI, 97.2-99.0\%). PLR and NLR were 10.6 (95\% CI, 7.1-15.7) and 0.33 (95\% CI, 0.2-0.6), respectively (Table 2).

\section{Characterization of False-Positive Patients}

The clinical features of the 37 non-pemphigus patients tested positive for intercellular antibodies were analyzed. These patients were followed for a median duration of 5.3 years (range, $0.7-16.9$ years), contributing 280.8 person-years.

Thirty patients $(81.1 \%)$ were eventually diagnosed clinically and immunopathologically with subepidermal autoimmune bullous diseases (SAIBD); 23 patients (62.2\%) with bullous pemphigoid (BP), of whom one patient had coexisting psoriasis, 4 patients (10.8\%) with mucous membrane pemphigoid, 2 patients (5.4\%) with linear IgA bullous dermatosis (had IgA intercellular antibodies), and one patient (2.7\%) with lichen planus pemphigoides. Apart from one patient with BP having dual intercellular and anti-basement membrane zone (BMZ) antibodies, all the remaining 29 (96.7\%) patients with SAIBD had isolated intercellular antibodies detected by IIF. The diagnosis of theses SAIBD was grounded on suggestive clinical presentation, compatible histopathology, and linear deposits of immunoreactants along the BMZ by DIF

TABLE 2 | Evaluation of indirect immunofluorescence immunoassay on monkey esophagus.

\begin{tabular}{|c|c|c|c|c|}
\hline & \multicolumn{2}{|c|}{ Pemphigus vulgaris } & \multicolumn{2}{|c|}{ Pemphigus foliaceus } \\
\hline & Value (\%) & $\begin{array}{c}95 \% \\
\text { confidence } \\
\text { interval }\end{array}$ & Value (\%) & $\begin{array}{c}95 \% \\
\text { confidence } \\
\text { interval }\end{array}$ \\
\hline Sensitivity & 87.1 & $76.2-94.3 \%$ & 69.0 & $49.2-84.7 \%$ \\
\hline Positive predictive value & 80.4 & $75.0-84.9 \%$ & 35.1 & $26.7-44.5 \%$ \\
\hline Negative predictive value & 96.0 & $94.2-97.3 \%$ & 98.3 & $97.2-99.0 \%$ \\
\hline Positive likelihood ratio & 13.4 & $9.8-18.4$ & 10.6 & $7.1-15.7$ \\
\hline Negative likelihood ratio & 0.14 & $0.1-0.2$ & 0.33 & $0.2-0.6$ \\
\hline
\end{tabular}




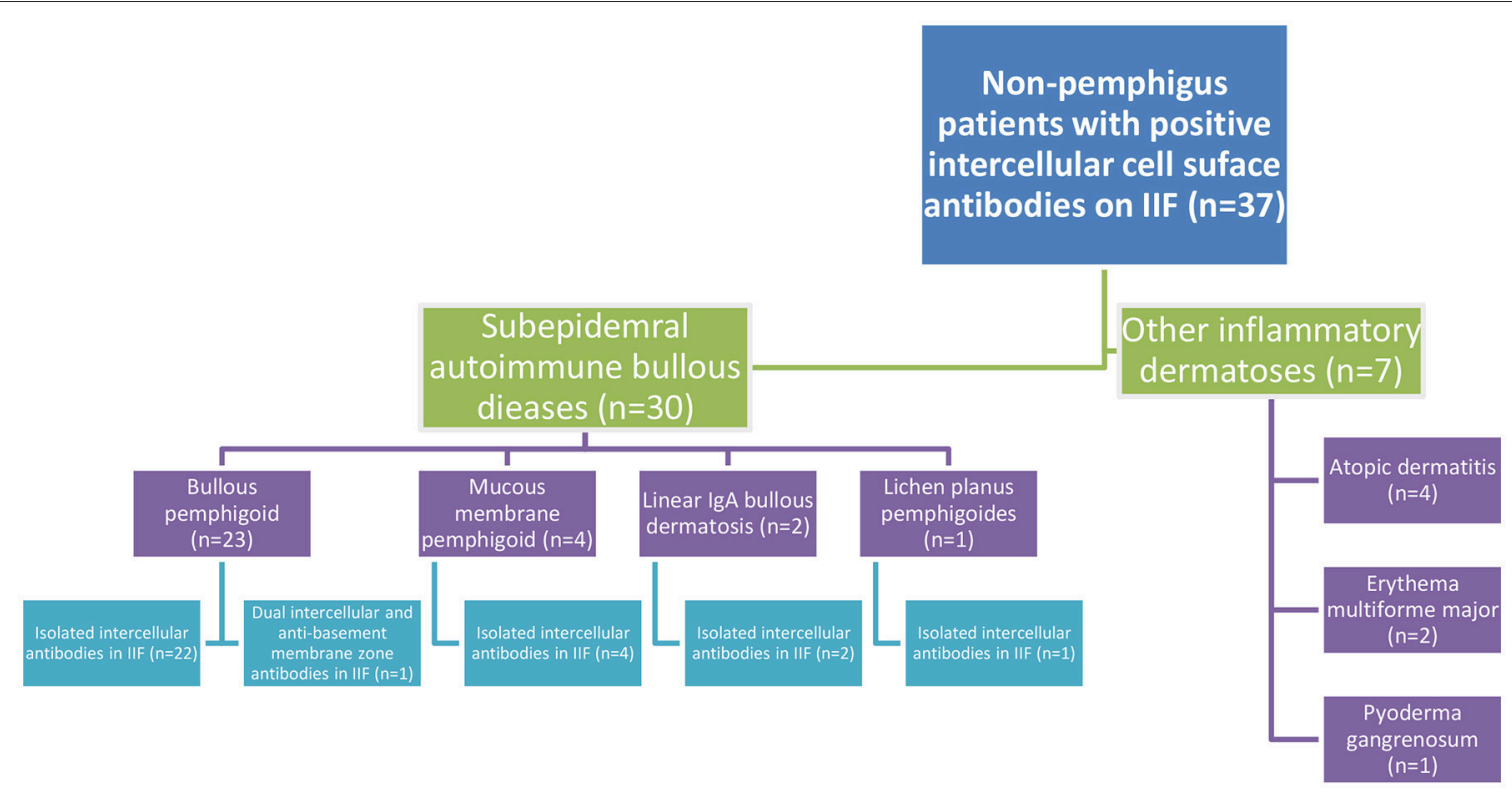

FIGURE 1 | Flowchart of the clinical characteristics of non-pemphigus patients tested positive for intercellular cell surface antibodies in IIF on monkey esophagus.

and/or the presence of circulating IgG antibodies against the immunodominant domain of BP180 (NC-16A) using ELISA (in cases of BP) (20). Intercellular tissue-bound antibodies was not detected in any of these patients, as DIF microscopy revealed isolated linear deposition along the BMZ in all 30 cases. Altogether, $7.0 \%(n=23)$ of all patients diagnosed with $(\mathrm{BP})$ in the same period $(n=328)$ were tested positive for IgG intercellular antibodies by IIF on monkey esophagus (Figure 1).

The remaining 7 patients were diagnosed clinically and histopathologically with various inflammatory dermatoses; 4 patients with atopic dermatitis, 2 patients with erythema multiforme major, and one patient with pyoderma gangrenosum (Figure 1).

None of the above 37 seropositive patients have subsequently developed pemphigus throughout the duration of follow up. Lesional biopsy specimens of 32 patients with the falsepositive results were reviewed, and no histopathological sign of acantholysis was detected.

\section{DISCUSSION}

The results of the current study indicate that IIF microscopy on monkey esophagus is a more sensitive test for the detection of circulating intercellular antibodies in PV than in PF. The specificity of the assay is high when used in routine practice, and the predictive value of a negative test is particularly reliable to exclude the diagnosis of pemphigus. Individuals tested positive for intercellular antibodies by IIF, without initial overt pemphigus, did not show an increased risk for developing pemphigus subsequently. None of the false-positive patients had histological evidence of acantholysis.
The positivity rate of IIF in previous cohorts varied depending on the epithelial substrate which the sera were incubated with. In a recent German study, intercellular epithelial staining on monkey esophagus by IIF was observed in 100.0 and $98.0 \%$ of 65 patients with $\mathrm{PV}$ and 50 patients with $\mathrm{PF}$, respectively (5). In an earlier British study, the diagnostic sensitivity of IIF on monkey esophagus was $100.0 \%$ in 20 patients with PV and $67.0 \%$ in 9 patients with PF (14). Jiao and Bysryn (4), examining 41 sera from PV patients and 22 sera from PF patients, had demonstrated that 87.0 and $86.0 \%$ of patients with PV and PF, respectively, were tested positive for intercellular antibodies by IIF when the assays were conducted on 2 different substrates simultaneously (monkey and guinea pig esophagus). In a small-scale study from Singapore, Ng PPL et al. (15) reported that all $13 \mathrm{PV}$ patients and 11 of 12 PF patients had positive IIF on monkey esophagus resulting in sensitivities of 100.0 and $91.7 \%$, respectively. In two previous serological studies performed in our center, the sensitivity of IIF on monkey esophagus was estimated at 96.0 and $81.0 \%$ for the diagnosis of PV in small cohorts of 25 and 32 patients, respectively $(18,21)$. Our study examined a 2 - to $10-$ fold larger number of patients and found that the sensitivity for PV (87.4\%) was lower than reported in most studies, whereas the sensitivity for $\mathrm{PF}$ (69.0\%) was within the range of previous studies $(4,14)$. The predictive value of a negative test in our study $(96.0 \%)$ was found to be particularly reliable to rule out a diagnosis of pemphigus. The large sample size in our study provides sufficient statistical power to exclude chance as the basis for the findings and sustains its external validity.

Several studies have estimated the the specificity of IIF by testing-healthy control subjects. A recent German study which examined 115 pemphigus patients estimated the specificity at 89.1\% (5). Another study comprising 33 Chinese pemphigus patients depicted that the specificity of this immunoassay was 
$91.8 \%$ (22). A similar specificity (94.8\%) was reported in a previous small-scale $(n=25)$ study from our center (18). A higher specificity of $100.0 \%$ was demonstrated by another serological study including 32 Israeli PV patients (21). Apart from the latter, the specificity revealed in our study $(93.5 \%)$ is comparable with that reported in other studies. It is noteworthy that all the aforementioned studies enrolled healthy control subjects in order to identify the specificity rates, most of them characterized by low pretest probability. Conversely, our study was undertaken in a real-life setting and consisted of individuals whose clinical presentation raised suspicion for autoimmune bullous diseases. Thus, the pretest probability was moderate to high in most control subjects. Our findings, therefore, should represent the real diagnostic value of this immunoassay in the everyday clinical practice more efficiently than studies recruiting healthy participants.

\section{Interpretation of Findings}

The higher sensitivity of this immunoassay for the diagnosis of $\mathrm{PV}$ is conceivable in light of the fact that monkey esophagus is a mucosal substrate with high expression of Dsg3, the main autoantigen in PV $(4,9,14)$. This substrate is less sensitive for PF patients with circulating anti-Dsg1 autoantibodies due to lower expression of Dsg1. IIF positivity depends on both the quantities of anti-Dsg1 and anti-Dsg3 antibodies in the test serum and the relative expression of Dsg1 and Dsg3 in the epithelial substrate. It was demonstrated that Dsg1-rich epithelial substrates like guinea pig esophagus and human skin were more sensitive than monkey esophagus for the diagnosis of $\operatorname{PF}(3,4)$. One study showed that the sensitivity of IIF on human skin was greater than on monkey esophagus in patients with PF, whereas the sensitivity of IIF on monkey esophagus was higher than on human skin in patients with PV (14). Thus, some authors claim that the combination of a Dsg1-rich substrate, such as guinea pig esophagus or human skin, and a Dsg3-rich substrate, such as monkey esophagus, is crucial prerequisite to increase the sensitivity of IIF when screening the sera of pemphigus patients. It is noteworthy that other authors reported conflicting results suggesting that the sensitivity of IIF on monkey esophagus was comparable in PV and PF patients $(5,15)$. The reason for this discrepancy is unknown.

The predominance of SAIBDs among patients with falsepositive assays may be ascribed to the "epitope spreading phenomenon"; a process in which a primary autoimmune or inflammatory cutaneous process may induce structural alterations in epidermal antigens $(23,24)$. The immune responses can spread over the disease course and recognize epitopes which are different from the original target. If it occurs in the same molecule, it is termed "intramolecular epitope spreading" (25), and if this immunological response involve epitopes on other proteins, it is then termed "intermolecular epitope spreading" (26). Regarding the relatively high false-positivity among patients with SAIBDs, it may postulated that intercellular cell-surface antigens that were previously concealed from the immune system became exposed, leading to the induction of a secondary autoimmune response that may be reflected by the production of non-pathogenic intercellular autoantibodies (24). A remarkable multicenter longitudinal study examined the profile of IgG autoantibody response to distinct BP180 and BP230 epitopes during the clinical course of $35 \mathrm{BP}$ patients (27). Epitope spreading events were detected in up to $50 \%$ of cases, mainly intramolecular epitope spreading events consisting of early IgG reactivity with extracellular epitopes, which was followed by IgG reactivity with intracellular epitopes of BP180. This study did not investigate the development of intermolecular epitope spreading against Dsg1/3 (27).

Sami et al. (28) presented 13 patients with an initial immunopathological diagnosis of BP who subsequently demonstrated coexistent serological features of both BP and $\mathrm{PV}$ and failed to respond to conventional systemic therapy. IIF using monkey esophagus as substrate revealed high levels of intercellular cell surface antibodies in all patients (7 in conjunction with anti-BMZ antibodies and 6 without antiBMZ antibodies). Additionally, all 13 patients had anti-Dsg3 antibodies and 9 had anti-Dsg1 antibodies on ELISA. The administration of intravenous immunoglobulin (IVIg) resulted in effective clinical response and the maintenance of prolonged clinical remission. In view of their findings, the authors recommended performing detailed serological re-evaluation and considering a dual diagnosis of $\mathrm{BP}$ and $\mathrm{PV}$ in patients with an initial diagnosis of BP who are nonresponsive to conventional therapy. Unlike these findings, the great majority of our "falsepositive" BP patients had isolated intercellular antibodies without simultaneous detection of anti-BMZ antibodies by IIF. While Sami et al. (28) attributed a pathogenic role for intercellular antibodies detected in patients with BP which supposedly rendered them more recalcitrant for conventional therapy, we did not find any distinct clinical features for BP patients with intercellular antibodies. In addition, histological review of lesional biopsy specimens did not reveal acantholysis, thus arguing against a pathogenetic role for intercellular antibodies detected in IIF in this subgroup.

In 2001, Sami and Ahmed (29) reviewed the literature and summarized 17 reported patients with mutual features of both BP and PV. Of whom, $83 \%$ had serum antibodies typical of PV. The present study demonstrated that this phenomenon occurred in $7 \%$ of BP patients. A notable case of 26 -years old woman presenting with tissue-bound and circulating antibodies suggestive of both pemphigoid gestationis (PG) and pemphigus in DIF and IIF, respectively, was reported (30). Clinically, she presented with erythematous eruption on the lower abdomen and thighs the day following delivery. The patient behaved clinically as a typical case of PG exhibiting a good response to moderate dose of oral corticosteroids, and lacked conventional clinical manifestation of pemphigus (30).

It was evidenced that IgG4 is the major subclass of autoantibodies in active pemphigus (31-33). Dsg-specific autoantibodies in pemphigus patients with active disease tend to preferentially associate with IgG4 subclass $(33,34)$. In both $\mathrm{PV}$ and PF, patients with active disease demonstrate Dsgreactive IgG4 and IgG1, while patients in remission and some healthy relatives of patients with pemphigus can demonstrate only anti-Dsg IgG1 (34-36). A recent study had revealed that serum IgG4, but not other IgG subclasses, was enriched in 
patients with pemphigus compared with unaffected individuals (32). Additionally, IgG4 depletion in PV sera diminished pathogenicity in a keratinocyte dissociation assay and depicted that affinity-purified IgG4 is more pathogenic than other serum IgG fractions (32). Another study found IgG4 to be the exclusive subclass that differentiates PV patient subgroups based on different disease morphologies and disease durations (33). Moreover, an IgG4-specific Dsg ELISA was verified to have greater sensitivity and specificity than a total IgG Dsg ELISA in identifying active disease in endemic PF, suggesting a more substantial clinical association of pathogenic antibodies with IgG4 rather than with other IgG subclasses (37). It is of great interest to explore whether false-positive patients had IgG4 anti-cell surface antibodies or, alternatively, IgG2 and IgG3 autoantibodies which have not been associated with a pathogenic role in pemphigus $(38,39)$. Given the retrospective data collection and the unavailability of the sera, characterization of the specific subclass of IgG antibodies could not be performed.

\section{Strengths and Limitations}

The sample size is large, and all the analyses were performed before the initiation of any immunosuppressive medications which could interfere with the results. Our study has some limitations to consider. First, the phenotypes of PV patients were not categorized according to the immunoserological profile. Second, although the immunoassays were performed in the same laboratory using the same substrate, at least 2 technicians analyzed the results of this subjective technique. The titers and the specific subclass of autoantibodies, as well as the specific pattern of deposition were not evaluated systematically in all patients. Thus, we could not investigate the association between autoantibodies levels and subclasses and the clinical characteristics.

\section{REFERENCES}

1. Stanley JR, Amagai M. Mechanisms of disease pemphigus, bullous impetigo, and the Staphylococcal scalded-skin syndrome. N Engl J Med. (2006) 355:1800-10. doi: 10.1056/NEJMra061111

2. Tsunoda K, Ota $T$, Saito M, Hata $T$, Shimizu A, Ishiko A, et al. Pathogenic relevance of IgG and IgM antibodies against desmoglein 3 in blister formation in pemphigus vulgaris. Am J Pathol. (2011) 179:795-806. doi: 10.1016/j.ajpath.2011.04.015

3. Sabolinski ML, Beutner EH, Krasny S, Kumar V, Huang J, Chorzelski TP, et al. Substrate specificity of anti-epithelial antibodies of pemphigus vulgaris and pemphigus foliaceus sera in immunofluorescence tests on monkey and guinea pig esophagus sections. J Invest Dermatol. (1987) 88:545-9. doi: 10.1111/1523-1747.ep12470131

4. Jiao D, Bystryn JC. Sensitivity of indirect immunofluorescence, substrate specificity, and immunoblotting in the diagnosis of pemphigus. J Am Acad Dermatol. (1997) 37:211-6. doi: 10.1016/S0190-9622(97)80127-2

5. van Beek N, Rentzsch K, Probst C, Komorowski L, Kasperkiewicz M, Fechner K, et al. Serological diagnosis of autoimmune bullous skin diseases: prospective comparison of the BIOCHIP mosaic-based indirect immunofluorescence technique with the conventional multi-step single test strategy. Orphanet J Rare Dis. (2012) 7:49. doi: 10.1186/1750-1172-7-49

6. Mitchell Sams W, Jordon RE. Correlation of pemphigoid and pemphigu s antibody titres with activity of disease. Br J Dermatol. (1971) 84:7-13. doi: 10.1111/j.1365-2133.1971.tb14190.x
It is noteworthy that a growing body of evidence accumulated in the last decade to signify the high sensitivity of Dsg 1 and Dsg 3-ELISA (40, 41). Many authors recommend utilizing this technique, available as mono-analyte or multi-analyte systems, as an easier technique. However, IIF is still widely used for the immunoserological diagnosis of pemphigus, specifically in low-income countries.

In conclusion, IIF microscopy on monkey esophagus is a more sensitive immunoassay for the detection of circulating intercellular autoantibodies in PV than in PF. The specificity of the assay is relatively high when used in real-life clinical settings, and the negative predictive value is particularly reliable to exclude the diagnosis of pemphigus. A notable proportion of patients with BP (7.0\%) showed false-positive circulating intercellular autoantibodies by IIF. None of the patients with false-positive results demonstrated a histological evidence for acantholysis or developed pemphigus during the follow-up duration, which argues against a pathogenic role of intercellular antibodies in this subgroup.

\section{ETHICS STATEMENT}

This retrospective, non-interventional study was approved by the institutional ethical board of Rambam Health Care Campus, waiving patient written informed consent.

\section{AUTHOR CONTRIBUTIONS}

KK substantial contributions to the conception or design of the work; or the acquisition, analysis, or interpretation of data for the work. KK and RB drafting the work or revising it critically for important intellectual content. KK and RB final approval of the version to be published.

7. Beutner EH, Chorzelski TP, Jablonska S. Immunofluorescence tests: clinical significance of sera and skin in bullous diseases. Int J Dermatol. (1985) 24:405-21. doi: 10.1111/j.1365-4362.1985.tb05507.x

8. Judd KP, Mescon H. Comparison of different epithelial substrates useful for indirect immunofluorescence testing of sera from patients with active pemphigus. J Invest Dermatol. (1979) 72:314-6. doi: 10.1111/1523-1747.ep12531752

9. Aksu D, Peksari Y, Arica IE, Gurgey E. Assessing the autoantibody levels in relation to disease severity and therapy response in pemphigus patients. Indian J Dermatol. (2010) 55:342-7. doi: 10.4103/0019-5154.74536

10. Fitzpatrick RE, Newcomer VD. The correlation of disease activity and antibody titers in pemphigus. Arch Dermatol. (1980) 116:285-90. doi: 10.1001/archderm.1980.01640270045011

11. Judd KP, Lever WF. Correlation of antibodies in skin and serum with disease severity in pemphigus. Arch Dermatol. (1979) 115:428-32. doi: 10.1001/archderm.1979.04010040006002

12. Acosta E, Gilkes JJH, Ivanyi L. Relationship between the serum autoantibody titers and the clinical activity of pemphigus vulgaris. Oral Surg Oral Med Oral Pathol. (1985) 60:611-14. doi: 10.1016/0030-4220(85)90363-9

13. Kridin K, Zelber-Sagi S, Khamaisi M, Cohen AD, Bergman R. Remarkable differences in the epidemiology of pemphigus among two ethnic populations in the same geographic region. J Am Acad Dermatol. (2016) 75:925-30. doi: 10.1016/j.jaad.2016.06.055

14. Harman KE, Gratian MJ, Bhogal BS, Challacombe SJ, Black MM. The use of two substrates to improve the sensitivity of indirect immunofluorescence 
in the diagnosis of pemphigus. Br J Dermatol. (2000) 142:1135-9. doi: 10.1046/j.1365-2133.2000.03538.x

15. Ng PPL, Thng STG, Mohamed K, Tan SH. Comparison of desmoglein ELISA and indirect immunofluorescence using two substrates (monkey oesophagus and normal human skin) in the diagnosis of pemphigus. Australas J Dermatol. (2005) 46:239-41. doi: 10.1111/j.1440-0960.2005.00191.x

16. Rowilson-Cunha P, Bystryn J-C. Sensitivity of indirect immunofluorescence and immunoblotting for the detection of intercellular antibodies in endemic pemphigus foliaceus (fogo selvagem). Int J Dermatol. (1999) 38:41-5. doi: 10.1046/j.1365-4362.1999.00568.x

17. Cunha PR, Bystryn JC, Medeiros EPL, de Oliveira JR. Sensitivity of indirect immunofluorescence and ELISA in detecting intercellular antibodies in endemic pemphigus foliaceus (Fogo Selvagem). Int J Dermatol. (2006) 45:9148. doi: $10.1111 /$ j.1365-4632.2006.02521.x

18. Sezin T, Avitan-Hersh E, Indelman M, Moscona R, Sabo E, Katz R, et al. Human amnion membrane as a substrate for the detection of autoantibodies in pemphigus vulgaris and bullous pemphigoid. Isr Med Assoc J. (2014) $16: 217-23$.

19. Mercaldo ND, Lau KF, Zhou XH. Confidence intervals for predictive values with an emphasis to case-control studies. Stat Med. (2007) 26:2170-2183. doi: $10.1002 /$ sim. 2677

20. Thoma-Uszynski S, Uter W, Schwietzke S, Hofmann SC, Hunziker T, Bernard P, et al. BP230- and BP180-specific auto-antibodies in bullous pemphigoid. $J$ Invest Dermatol. (2004) 122:1413-22. doi: 10.1111/j.0022-202X.2004.22603.x

21. Zagorodniuk I, Weltfriend S, Shtruminger L, Sprecher E, Kogan O, Pollack S, et al. A comparison of anti-desmoglein antibodies and indirect immunofluorescence in the serodiagnosis of pemphigus vulgaris. Int $J$ Dermatol. (2005) 44:541-4. doi: 10.1111/j.1365-4632.2004.02541.x

22. Zhou $\mathrm{T}$, Fang $\mathrm{S}$, Li C, Hua H. Comparative study of indirect immunofluorescence, enzyme-linked immunosorbent assay, and the Tzanck smear test for the diagnosis of pemphigus. J Oral Pathol Med. (2016) 45:786-790. doi: 10.1111/jop.12439

23. Ohyama B, Nishifuji K, Chan PT, Kawaguchi A, Yamashita T, Ishii N, et al. Epitope spreading is rarely found in pemphigus vulgaris by large-scale longitudinal study using desmoglein 2-based swapped molecules. J Invest Dermatol. (2012) 132:1158-68. doi: 10.1038/jid.2011.448

24. Chan LS, Vanderlugt CJ, Hashimoto T, Nishikawa T, Zone JJ, Black MM, et al. Epitope spreading: lessons from autoimmune skin diseases. J Invest Dermatol. (1998) 110:103-109. doi: 10.1046/j.1523-1747.1998.00107.x

25. Lehmann P V., Forsthuber T, Miller A, Sercarz EE. Spreading of T-cell autoimmunity to cryptic determinants of an autoantigen. Nature (1992) 358:155-157. doi: 10.1038/358155a0

26. Steinman L, Conlon P. Viral damage and the breakdown of self-tolerance. Nat Med. (1997) 3:1085-87. doi: 10.1038/nm1097-1085

27. Di Zenzo G, Thoma-Uszynski S, Calabresi V, Fontao L, Hofmann SC, Lacour JP, et al. Demonstration of epitope-spreading phenomena in bullous pemphigoid: Results of a prospective multicenter study. J Invest Dermatol. (2011) 131:2271-80. doi: 10.1038/jid.2011.180

28. Samia N, Bhol KC, Beutner EH, Plunkett RW, Leiferman KM, Ahmed AR. Diagnostic features of pemphigus vulgaris in patients with bullous pemphigoid - Molecular analysis of autoantibody profile. Dermatology (2002) 204:108-17. doi: 10.1159/000051827

29. Sami N, Ahmed AR. Dual diagnosis of pemphigus and pemphigoid. Dermatology (2001) 202:293-301. doi: 10.1159/000051661

30. Vaughan Jones SA, Bhogal BS, Black MM, Clement M, Hashimoto T, Nishikawa T. A typical case of pemphigoid gestationis with a unique pattern of intercellular immunofluorescence. Br J Dermatol. (1997) 136:245-48. doi: 10.1046/j.1365-2133.1997.d01-1179.x

31. Dainichi T, Chow Z, Kabashima K. IgG4, complement, and the mechanisms of blister formation in pemphigus and bullous pemphigoid. J Dermatol Sci. (2017) 88:265-70. doi: 10.1016/j.jdermsci.2017.07.012

32. Funakoshi T, Lunardon L, Ellebrecht CT, Nagler AR, O'Leary CE, Payne AS. Enrichment of total serum IgG4 in patients with pemphigus. Br J Dermatol. (2012) 167:1245-53. doi: 10.1111/j.1365-2133.2012.11144.x

33. Dhandha MM, Seiffert-Sinha K, Sinha AA. Specific immunoglobulin isotypes correlate with disease activity, morphology, duration and HLA association in Pemphigus vulgaris. Autoimmunity (2012) 45:516-26. doi: 10.3109/08916934.2012.702811

34. Kricheli D, David M, Frusic-Zlotkin M, Goldsmith D, Rabinov M, Sulkes J, et al. The distribution of pemphigus vulgaris-IgG subclasses and their reactivity with desmoglein 3 and 1 in pemphigus patients and their first-degree relatives. $\mathrm{Br} J$ Dermatol. (2000) 143:337-42. doi: $10.1046 / j .1365-2133.2000 .03659 . x$

35. Rock B, Martins CR, Theofilopoulos AN, Balderas RS, Anhalt GJ, Labib $\mathrm{RS}$, et al. The pathogenic effect of IgG4 autoantibodies in endemic pemphigus foliaceus (fogo selvagem). N Engl J Med. (1989) 320:1463-9. doi: 10.1056/NEJM198906013202206

36. Warren SJP, Arteaga LA, Rivitti EA, Aoki V, Hans-Filho G, Qaqish $\mathrm{BF}$, et al. The role of subclass switching in the pathogenesis of endemic Pemphigus Foliaceus. J Invest Dermatol. (2003) 120:1-5. doi: 10.1046/j.1523-1747.2003.12017.x

37. Qaqish BF, Prisayanh P, Qian Y, Andraca E, Li N, Aoki V, et al. Development of an IgG4-based predictor of endemic pemphigus foliaceus (fogo selvagem). J Invest Dermatol. (2009) 129:110-118. doi: 10.1038/jid.2008.189

38. Futei $Y$, Amagai $M$, Ishii $K$, Kuroda-Kinoshita $K$, Ohya $K$, Nishikawa T. Predominant IgG4 subclass in autoantibodies of pemphigus vulgaris and foliaceus. J Dermatol Sci. (2001) 26:55-61. doi: 10.1016/S0923-1811(00)00158-4

39. Torzecka JD, Wozniak K, Kowalewski C, Waszczykowska E, SysaJedrzejowska A, Pas $\mathrm{HH}$, et al. Circulating pemphigus autoantibodies in healthy relatives of pemphigus patients: Coincidental phenomenon with a risk of disease development? Arch Dermatol Res (2007) 299:239-43. doi: $10.1007 /$ s00403-007-0760-y

40. Schmidt E, Dähnrich C, Rosemann A, Probst C, Komorowski L, Saschenbrecker S, et al. Novel ELISA systems for antibodies to desmoglein 1 and 3: Correlation of disease activity with serum autoantibody levels in individual pemphigus patients. Exp Dermatol. (2010) 19:458-63. doi: $10.1111 / j .1600-0625.2010 .01069 . x$

41. van Beek N, Dähnrich C, Johannsen N, Lemcke S, Goletz S, Hübner F, et al. Prospective studies on the routine use of a novel multivariant enzyme-linked immunosorbent assay for the diagnosis of autoimmune bullous diseases. J Am Acad Dermatol. (2017) 76:889-94.e5. doi: 10.1016/j.jaad.2016.11.002

Conflict of Interest Statement: The authors declare that the research was conducted in the absence of any commercial or financial relationships that could be construed as a potential conflict of interest.

Copyright $\odot 2018$ Kridin and Bergman. This is an open-access article distributed under the terms of the Creative Commons Attribution License (CC BY). The use, distribution or reproduction in other forums is permitted, provided the original author(s) and the copyright owner(s) are credited and that the original publication in this journal is cited, in accordance with accepted academic practice. No use, distribution or reproduction is permitted which does not comply with these terms. 